\title{
Coffee consumption and reduced risk of hepatocellular carcinoma: findings from the Singapore Chinese Health Study
}

\author{
Shane Johnson, \\ The Masonic Cancer MMC 806, University of Minnesota, 425 East River Road, Minneapolis, MN \\ 55455, USA; Division of Epidemiology and Community Health, School of Public Health, University \\ of Minnesota, Minneapolis, MN, USA
}

\section{Woon-Puay Koh,}

Department of Epidemiology and Public Health, Yong Loo Lin School of Medicine, National University of Singapore, Kent Ridge, Singapore

\section{Renwei Wang,}

The Masonic Cancer MMC 806, University of Minnesota, 425 East River Road, Minneapolis, MN 55455, USA

\section{Sugantha Govindarajan, \\ Department of Clinical Pathology, Rancho Los Amigos National Rehabilitation Center, Keck School of Medicine, University of Southern California, Los Angeles, CA, USA}

Mimi C. Yu, and

The Masonic Cancer MMC 806, University of Minnesota, 425 East River Road, Minneapolis, MN 55455, USA

\section{Jian-Min Yuan}

The Masonic Cancer MMC 806, University of Minnesota, 425 East River Road, Minneapolis, MN 55455, USA; Division of Epidemiology and Community Health, School of Public Health, University of Minnesota, Minneapolis, MN, USA

\section{Abstract}

Background-Coffee consumption has been associated with reduced markers of hepatic cell damage, reduced risk of chronic liver disease, and cirrhosis across a variety of populations. Data on the association between coffee consumption and risk of hepatocellular carcinoma (HCC), especially in high-risk populations, are sparse.

Methods-This study examines the relationship between coffee and caffeine consumption, and the risk of developing HCC within the Singapore Chinese Health Study, a prospective cohort of 63,257 middle-aged and older Chinese men and women, a relatively high-risk population for HCC. Baseline data on coffee consumption and other dietary and lifestyle factors were collected through inperson interviews at enrollment between 1993 and 1998.

Results-As of 31 December 2006, 362 cohort participants had developed HCC. High levels of coffee or caffeine consumption were associated with reduced risk of HCC ( $p$ for trend $<0.05$ ). Compared with non-drinkers of coffee, individuals who consumed three or more cups of coffee

\footnotetext{
(C) Springer Science+Business Media B.V. 2011

Correspondence to: Jian-Min Yuan.

jyuan@umn.edu.

Electronic supplementary material The online version of this article (doi:10.1007/s10552-010-9725-0) contains supplementary material, which is available to authorized users.
} 
per day experienced a statistically significant $44 \%$ reduction in risk of HCC (hazard ratio 0.56, 95\% confidence interval, $0.31-1.00, p=.049)$ after adjustment for potential confounders and tea consumption.

Conclusion-These data suggest that coffee consumption may reduce the risk of developing HCC in Chinese in Singapore.

\section{Keywords}

Coffee; Caffeine; HCC; Hepatocellular; Liver cancer; GGT

\section{Introduction}

Although rare in North America and Europe, primary liver cancer is a common malignancy worldwide. It is the fifth most common cancer and third most common cause of cancer death in men, and the eighth most common cancer and sixth most common cause of cancer death in women, accounting for approximately $6 \%$ of all newly diagnosed cancer cases worldwide [1].

The dominant form of primary liver cancer is hepatocellular carcinoma (HCC). Chronic infection with hepatitis B virus (HBV) is by far the most important risk factor for $\mathrm{HCC}$ in humans. It is the primary cause of HCC in high-risk areas, including China and Africa. Chronic infection with hepatitis $\mathrm{C}$ virus ( $\mathrm{HCV}$ ) has an increasingly prominent role in the development of HCC in countries such as the United States where the rate of infection with HBV is relatively low. Other environmental risk factors for HCC include dietary aflatoxin, excessive alcohol intake, cigarette smoking, obesity, and diabetes [2].

Recent studies also suggest a protective effect of coffee and coffee constituents on liver function. Caffeine has a strong antioxidative property [3], inhibits the cell proliferation of liver cancer cells [4], and reduces the incidence and multiplicity of carcinogen-induced liver cancer in rats [5]. Coffee consumption has been associated with reduced levels of gammaglutamyltransferase (GGT), alanine aminotransferase (ALT), and aspartate aminotransferase (AST), biomarkers of liver cell injury and inflammation [6].

In addition to caffeine, unfiltered coffee contains large quantities of kahweol and cafestol [7]. Experimental studies have shown that kahweol and cafestol can reduce the genotoxicity of aflatoxin $B_{1}$ in hepatic cell lines [8], an established human hepatocarcinogen [9]. Both kahweol and cafestol also can induce enzymes in the liver that enhance the detoxification of other potential carcinogens such as N-nitrosodimethylamine (NDMA) and 2-amino-1methyl-6-phenylimidazopyridine (PhIP) [10].

Numerous epidemiologic studies have examined the association between coffee consumption and risk of HCC [11-18]. Overall, these studies showed an inverse association between coffee consumption and HCC risk [19, 20]. These studies were conducted largely in Japanese and Italian populations. There is lack of data on the potential protective effects of coffee consumption on the development of HCC in Chinese populations that are at high risk of $\mathrm{HCC}$ and have different exposure to environmental risk factors. For example, the incidence rate of HCC in Chinese men in Singapore was 21.2 per 100,000, more than 5 times that of their white counterparts in United States [21]. Using the database of the Singapore Chinese Health Study, we prospectively examined the relation of coffee and other caffeine containing beverages with the risk of developing HCC. 


\section{Materials and methods}

\section{Study population}

The design of the Singapore Chinese Health Study has been described [22]. Permanent resident or citizen of Singapore of Chinese origin speaking Hokkien or Cantonese dialect between ages of 45 and 74 years residing in government-built housing estates were sought for their participation of the study. Between April 1993 and December 1998, 63,257 subjects (about $85 \%$ of eligible subjects approached) were recruited. The study was approved by the Institutional Review Boards of the National University of Singapore and the University of Minnesota.

\section{Baseline exposure assessment}

At recruitment, an in-person interview was conducted in the subject's home by a trained interviewer using a structured questionnaire, which covered demographics, lifetime use of tobacco, current physical activity, menstrual/reproductive history (for women), occupational exposure, medical history, and family history of cancer. Information on current diet, including alcohol, coffee, and tea consumption, was assessed via a 165 -item food frequency questionnaire that has been validated against a series of 24-h dietary recall interviews [22] and selected biomarker studies [23] conducted on random subsets of cohort participants.

Levels of caffeine intake were derived from self-report of coffee and tea intake, the two major sources of caffeine in this population. Our baseline questionnaire asked for information on consumption of coffee, black tea, and other types of tea (this included green tea). For each of these beverages, participants were asked to choose their level of consumption from the following pre-defined categories: never or hardly ever, 1 to 3 times a month, once a week, 2 to 3 times a week, 4 to 6 times a week, once a day, 2 to 3 times daily, 4 to 5 times daily, and 6 or more times daily. The majority of caffeine consumed was from coffee $(82 \%)$ and black tea $(13 \%)$. Green tea was a minor contributor to caffeine $(<5 \%)$ in the study population.

\section{Case ascertainment}

Incident cases of cancer and death among cohort members were identified by linkage analysis of the cohort master files with the datasets of the population-based Singapore Cancer Registry and Singapore Registry of Births and Deaths. The nationwide cancer registry has been in place since 1968 and has been shown to be comprehensive in its recording of incident cancer cases [24]. As of April 2008, only 27 cases were known to be lost to follow-up due to migration out of Singapore. This suggests that emigration is negligible among the cohort participants. As of 31 December 2006, 362 cohort participants who were free of cancer at baseline had developed HCC. One hundred and fourteen (31.5\%) HCC cases were diagnosed histologically, and their diagnoses were confirmed via manual review of pathology reports by a medically trained research staff. Two hundred and thirtyfive $(65.0 \%)$ were diagnosed on the basis of elevation in serum a-fetoprotein in conjunction with clinical and radiologic evidence consistent with HCC. Thirteen (3.5\%) cases were identified through death certificates.

\section{Hepatitis $B$ and $C$ serology}

Beginning in April 1994, a random 3\% sample of cohort participants were asked to provide blood or buccal cells, and spot urine samples. Eligibility for this biospecimen subcohort was extended to all surviving cohort participants starting in January 2000. By April 2005, all surviving cohort subjects had been contacted for biospecimen donation. Samples were obtained from 32,535 subjects, representing a consent rate of about $60 \%$. 
Of the 362 incident HCC patients among the entire cohort, 92 were from the biospecimen subcohort that provided a blood sample at baseline. For each case, three control subjects individually matched to the index case by gender, dialect group (Hokkien, Cantonese), age at enrollment ( \pm 2 years), date of baseline interview ( \pm 1 year), and date of biospecimen collection ( \pm 6 months) were randomly selected from the biospecimen subcohort who were alive and free of cancer on the date of cancer diagnosis of the index case. The assays used for testing serologic markers of $\mathrm{HBV}$ and $\mathrm{HCV}$ infections have been described previously [25]. Briefly, we tested all study samples for the presence of hepatitis B surface antigen (HBsAg) in plasma using commercialized kits (AUSRIA, Abbott Laboratories, North Chicago, IL), and negative samples were further tested for the presence of antibodies to the hepatitis B core antigen (anti-HBc) and antibodies to the hepatitis B surface antigen (antiHBs) using standard testing kits (Corab, Abbott Laboratories, North Chicago, IL). All samples were tested for the presence of antibodies to the hepatitis C virus (anti-HCV) in plasma using the ELISA version 2.0 kit manufactured by Ortho Diagnostic Systems, with confirmation of positive samples using RIBA version 2.0 (Chiron, Emeryville, CA). Plasma samples were tested blindly, identified only by codes without regard to case-control status.

\section{Data analysis}

We excluded 1,936 individuals with a history of invasive cancer of all sites (except nonmelanoma skin cancer) as well as superficial (i.e., non-invasive) bladder cancer at baseline interview in order to avoid the potential effects of changes in lifestyle factors, including coffee drinking, in patients following cancer diagnosis. The present analysis included 61,321 subjects. The distributions of selected demographic characteristics and lifestyle factors between HCC cases and non-HCC subjects, or by levels of coffee consumptions were examined by chi-square test (for categorical variables) or by t-test or analysis of variance (for continuous variables).

For each subject, person-years of follow-up, stratified simultaneously by calendar time and age at recruitment, were counted from the date of recruitment to the date of diagnosis of HCC, death, or 31 December 2006, whichever occurred first. Proportional hazards (Cox) regression was used to analyze the associations between coffee (or caffeine) consumption and the risk of developing HCC. The strength of association was measured by hazard ratio (HR) and its 95\% confidence interval (CI) and $p$ value. All Cox regression models included age at recruitment (years), gender, dialect group (Hokkien, Cantonese), year of recruitment (1993-1995, 1996-1998), body mass index ( $\_4 \mathrm{~kg} / \mathrm{m}^{2},>24 \mathrm{~kg} / \mathrm{m}^{2}$ ), level of education (no formal schooling, primary school, or secondary school or higher), cigarette smoking (never, former, or current smoker), consumption of alcoholic beverages (non-drinker, $<7$, or $7+$ drinks per week), frequency of black and green tea consumption (none, monthly, weekly, or daily), and history of diabetes (no, yes).

To further adjust for the potential confounding effect of HBV/HCV infection on the coffee/ caffeine-HCC risk association, we conducted analyses for the association between coffee/ caffeine and HCC risk on 92 HCC patients and their 276 matched control subjects using the conditional logistic regression methods [26]. In addition to the variables for serological status of HBV and HCV (negative, positive), we included all potential confounders described above in all logistic regression models. Odds ratios (ORs) and their corresponding 95\% confidence interval (CIs) and $p$ values were used to measure the strength of the association between consumption of coffee consumption and HCC risk.

Statistical computing was conducted using SAS version 9.1 statistical software. All $p$ values quoted are two-sided, and $p<0.05$ were considered statistically significant. 


\section{Results}

Of the 61,321 individuals in the cohort, $18.5 \%$ were coffee non-drinkers, $11.1 \%$ consumed $<1$ cup/day (defined as light consumption), 36.0\% consumed 1 to <2 cups/day (medium consumption), and $34.4 \%$ consumed $\geq 2$ cups/day (heavy consumption). Men consumed more coffee than women, so did Hokkiens than Cantonese. Compared with coffee nondrinkers, heavy coffee drinkers consumed more cigarettes and alcoholic beverages. Coffee consumption was inversely associated with black tea and green tea consumption. Subjects with a history of diabetes drank less coffee (see details in Supplemental Table 1).

The mean age ( \pm standard deviation) of the 362 patients with $\mathrm{HCC}$ at diagnosis was 67.7 $( \pm 7.9)$ years. The mean time interval between baseline interview and cancer diagnosis was 6.4 years (range, 1 month to 13 years). Men were three times more likely to have HCC than women. Hokkiens accounted for $62.7 \%$ of HCC cases. HCC patients consumed more alcoholic beverages and cigarettes and were more likely to have a history of diabetes than those who remained free of HCC (Supplemental Table 2).

Coffee and caffeine consumption were inversely associated with the risk of developing HCC (Table 1). The risk of HCC decreased with increasing number of cups of coffee consumed ( $p$ for trend $=0.05$ ). Compared with coffee nondrinkers, individuals who consumed $\geq 3$ cups/ day had a statistically significant $44 \%$ reduction in risk of HCC $(p=0.05)$ after adjustment for multiple potential confounders. Since coffee is the main source of caffeine in the study population, a similar inverse association between the amount of caffeine consumed and risk of HCC was observed. Compared with the lowest quartile, HRs (95\% CIs) for the 2nd, 3rd, and 4th quartile of caffeine intake were $1.09(0.82-1.46), 0.82$ (0.61-1.11), and 0.73 (0.541.00 ), respectively ( $p$ for trend $=0.02$ ).

Given that both coffee and tea contain caffeine, we further analyzed the relationship of HCC risk with coffee/caffeine intake in combination with tea intake. Within each intake level of green tea, black tea, or any tea (i.e., both green and black tea combined), high intake of coffee and caffeine was associated with a 20-50\% decreased risk of HCC compared with low intake of coffee/caffeine, respectively (Table 2). Conversely, within each intake category of coffee or caffeine, drinkers of either green tea or black tea had a comparable risk of HCC with those who did not consume green tea, black tea, or any tea.

The association between caffeine intake and HCC risk was further examined in subgroups stratified by gender, body mass index, cigarette smoking status, alcohol drinking, and history of diabetes. The inverse association between caffeine intake and HCC risk in women was comparable to that in men although the trend test in the former was not statistically significant due to small sample size (Supplemental Table 3). The inverse caffeine-HCC risk associations were comparable between smokers and non-smokers, between alcohol drinkers and non-alcohol drinkers, between individuals with high $\left(\geq 24 \mathrm{~kg} / \mathrm{m}^{2}\right)$ and low body mass index $\left(<24 \mathrm{~kg} / \mathrm{m}^{2}\right)$ (all Ps for interaction $\left.>0.55\right)$. The inverse association between caffeine consumption and $\mathrm{HCC}$ risk was stronger in diabetic patients than that in subjects without a history of diabetes, but the difference between the two groups was not statistically significant $(p$ for interaction $=0.11$ ). Subgroup analysis examining the relationship between coffee consumption and $\mathrm{HCC}$ yielded results similar to that observed when examining the caffeine-HCC association. The protective effect of coffee consumption was, again, strongest among diabetics.

We further examined the association between coffee/caffeine consumption and HCC risk after adjustment for serology of HBV and HCV. This analysis was conducted on $92 \mathrm{HCC}$ patients and 276 individually matched controls with known serology of HBsAg, anti-HBc, and anti-HCV. As shown in Table 3, the point estimates of relative risk of HCC associated 
with high consumption of coffee or caffeine in this subgroup were very similar to those based on the entire cohort. However, all ORs were not statistically significant due to small number of HCC cases included in this subgroup analysis. Further adjustment for serology of $\mathrm{HBV}$ and HCV did not materially alter the inverse association between coffee/caffeine consumption and risk of HCC, suggesting that the observed inverse association between coffee/caffeine consumption and HCC risk would be less likely to be confounded by HBV and/HCV infection.

\section{Discussion}

In this prospective analysis of a large-scale, population-based cohort study of Chinese in Singapore, a statistically significant inverse association between consumption of coffee and the risk of developing HCC was observed. Compared with coffee non-drinkers, drinkers of coffee with three or more cups per day experienced a statistically significant $44 \%$ decreased risk of HCC. Findings of the present study in a high-risk population for $\mathrm{HCC}$ are consistent with previous epidemiological studies conducted in low- to intermediate-risk populations and further support the hypothesis that coffee or specific constituents in the coffee may protect against the development of HCC in humans.

The inverse association between coffee consumption and HCC risk may arise from the fact that subjects with a broad spectrum of liver disorders and cirrhosis choose to reduce their coffee consumption. However, previous studies found that the inverse association between coffee consumption and risk of HCC was present in subjects with or without chronic liver disease [19]. One cohort study in Japan reported a statistically significant inverse association between coffee consumption and risk of HCC among the entire cohort. When HCC cases were stratified by HBV and/or HCV serology, the inverse coffee-HCC risk association was present for HCC cases that tested negative for both HBV and HCV [13]. In the present study, we determined HBV and HCV serology in a subset of HCC cases and matched control subjects. Although the overall association between coffee/caffeine consumption and HCC risk among this subset of individuals with known HBV/HCV serologic status was no longer statistically significant, primarily due to the small number of HCC patients who donated pre-diagnosed blood samples for the test of hepatitis serology, further adjustment for HBV and HCV serology did not alter the inverse association between coffee/caffeine consumption and HCC risk, suggesting that infection with HBV and/or HCV or preexisting chronic liver disease would be less likely to confound the observed inverse coffee-HCC risk association.

Tea contains not only caffeine but also polyphenols, both of which show antioxidative properties. Coffee drinkers of the present study population consumed less green or black tea. Our detailed analysis revealed that the reduced risk of $\mathrm{HCC}$ was mainly associated with consumption of coffee, but not with tea, suggesting that unique constituents in coffee may exert a protective effect.

Besides HCC, coffee consumption has been inversely related to serum ALT activity (a specific marker of liver injury), GGT activity (a marker of liver cirrhosis risk), cirrhosis, and other chronic liver disease [7, 27]. Given that cirrhosis is a hallmark of the liver disease progression towards HCC, these data have lent biological plausibility to the observed coffeeHCC risk association.

The biologic mechanism behind coffee consumption and HCC risk reduction is unclear. Coffee contains more than one thousand chemical compounds including caffeine, diterpenes (cafestol and kahweol), and chlorogenic acids. In one experimental study, caffeine in drinking water significantly reduced the incidence and multiplicity of chemical-induced 
liver tumors in rats [5]. The risk of cirrhosis was not associated with consumption of caffeinated, non-coffee beverages, but with consumption of coffee [28]. Furthermore, decaffeinated coffee has been shown to possess a similar effect as caffeinated coffee on altering phase I and phase II enzymes that probably participate in metabolic activation and detoxification of potential hepatocarcinogens, respectively (see below) [29]. These data suggest that compounds other than caffeine in coffee may exert beneficial effects on the liver.

Numerous studies have suggested that cafestol and kahweol may exert antihepatocarcinogenesis effects. In animal and cell line models, these diterpenes have been shown to significantly reduce the mRNA and protein levels of cytochrome P450 (CYP) isoenzymes $1 \mathrm{~A} 1,1 \mathrm{~A} 2,3 \mathrm{~A} 2,2 \mathrm{~B} 1,2 \mathrm{~B} 2$, and $2 \mathrm{C} 11$, along with the reduced formation of aflatoxin $\mathrm{B}_{1}\left(\mathrm{AFB}_{1}\right)$-DNA adducts [30], an established risk factor for HCC in humans[9]. In addition, cafestol and kahweol can upregulate phase II detoxifying enzymes including glutathione S-transferase (GST). The most striking effect identified is a strong dosedependent induction of the GST pi subunit Yp and Yc2 in the liver at the mRNA and protein levels following long-term treatment with diet containing cafestol and kahweol [8]. Moreover, the increased GST expression is dependent on the continuous presence of cafestol and kahweol in the diet; GST returned to the baseline level following removal of cafestol and kahweol from diet [9]. GST Yc2 is known to efficiently conjugate $\mathrm{AFB}_{1}-8,9-$ epoxide[8], the most genotoxic metabolite of $\mathrm{AFB}_{1}[31]$.

The presence of cafestol and kahweol in coffee is dependent on the method of preparation. Scandinavian boiled, French press (i.e., plunger or cafetière coffee), and Turkish/Greek coffees have the highest amounts of the diterpenes, espressos intermediate amounts, and instant and drip-filtered coffees have negligible amounts (paper filters retain the lipid fraction released during brewing) [7]. In Singapore, dark roasted coffee is commonly prepared by boiling in a muslin bag, a method presumed to preserve cafestol and kahweol levels. Due to the hypercholesterolemic effect of these diterpenes, the positive association between coffee intake and plasma cholesterol in our cohort supports our assumption that the diterpenes are present in the coffee commonly consumed by cohort subjects [32].

Chlorogenic acid present in coffee has shown twice the total antioxidant capacity of ascorbic acid over the same concentration range, indicating that chlorogenic acid can effectively scavenge reactive oxygen species, which have mutagenic and tumor-promoting effects [33]. Chlorogenic acid also increased enzymatic activities of GST and suppressed the chemicalinduced neoplastic transformation of cancer cells [32]. Rats fed a diet with instant coffee, which has no diterpenes, with or without caffeine showed a dose-dependent increase in mRNA and protein expression of GST enzymes involved in cellular antioxidant defenses, along with a protection against both genotoxicity and cytotoxicity of $\mathrm{AFB}_{1}$ [29]. These data suggest that ingredients of coffee other than diterpenes and caffeine may also play a role in the protection against the development of HCC.

Tea consumption, particularly green tea consumption, has been suggested as having protective effects against the development of cancer at a number of sites. Green tea polyphenols show antioxidative properties [34], which may protect against oxidative damage in humans [35]. Several epidemiologic studies have examined the association between green tea consumption and HCC risk [13, 16, 36-39]. Most of those studies reported a null association [13,16, 37-39]. A recent report from a prospective cohort study in Japan found a dose-dependent inverse association between green tea consumption and liver cancer incidence in women, but not in men. Women consuming 25 cups green tea per day experienced only half the risk that for women with $<1$ cup green tea per day [36]. We did not find a statistically significant association between consumption of either green tea or 
black tea at least on a weekly basis and HCC risk in the present study. To control for potential confounding effect of coffee on the tea-HCC risk association, we analyzed data cross-classified by consumption of green/black tea and coffee. The null tea-HCC risk association was present in both low (B2 cups/day) and high coffee drinkers ( $>2$ cups/day), whereas high consumption of coffee was associated with reduced risk of HCC in tea drinkers and non-drinkers. Our null findings on tea and HCC risk, consistent with most of epidemiological studies, could be due to the low consumption of green tea in our study population (12.4\% of cohort participants were daily drinkers who, on average, consumed 2.0 cups of green tea per day) compared with the study subjects of the Japanese cohort of which subjects consuming $\geq 1$ and $\geq 5$ cups green tea per day accounted for $73 \%$ and $30 \%$, respectively [36].

Potential limitations of this study include the lack of HBV and HCV status for all cohort participants, though some effort was made to address this using a nested case-control study. Additionally, participants in the cohort were not measured for the amount of liver damage present at baseline, making it difficult to address what effect this might have on the association between coffee consumption and risk of HCC. Finally, the number of observed cases is still relatively small in this cohort when compared to some others. This is particularly true among those where HBV/HCV serology is available. Given a small proportion of HCC cases with available prediagnostic blood samples, potential selection bias is a concern. However, we examined and found comparable distributions between HCC cases with and those without known HBV and HCV serology in coffee consumption (1.23 vs. 1.30 cups per day, $p=.59$ ), body mass index ( $\left.23.9 \mathrm{vs.} 23.7 \mathrm{~kg} / \mathrm{m}^{2}, p=.64\right)$, level of education ( $28.9 \%$ vs. $23.6 \%$ of secondary school or higher, $p=.51$ ), alcohol consumption ( $9.6 \%$ vs. $10.0 \%$ of $\geq 7$ drinks per week, $p=.17$ ), and history of diabetes ( $22.9 \%$ vs. $18.2 \%$, $p=.35$ ). These data indicate that patients with HCC of the nested case-control study were a representative sample of the HCC patients of the entire cohort.

The strengths of this study include its prospective nature. In all participants, exposure factors were measured before the diagnosis of disease, allowing us to analyze the data in a temporal manner. The relatively high consumption of both coffee and tea in Singaporean Chinese provided a unique setting to test whether coffee or tea or both exert protective effect on HCC risk in this high-risk population given that coffee is a less popular drink for Chinese in mainland China or elsewhere. The presence of an inverse association between coffee/ caffeine and HCC risk among non-drinkers of any tea confirmed the protective effect of coffee against the development of HCC, whereas tea did not show any significantly protective effect. The completeness of ascertainment of HCC cases among the cohort through the nationwide cancer registry in Singapore minimized the selection bias given that very few cohort participants emigrated from Singapore. Finally, the comprehensive questionnaire that asked for demographic, lifestyle, and other environmental exposures allowed for more thorough adjustment and less residual confounding effect on the observed coffee-HCC risk association.

In summary, high consumption of coffee/caffeine was associated with a statistically significant, reduced risk of HCC among Singaporean Chinese. Further studies are warranted to clarify the protective role of specific constituents in coffee in the development of HCC.

\section{Supplementary Material}

Refer to Web version on PubMed Central for supplementary material. 


\section{Acknowledgments}

We thank Siew-Hong Low of the National University of Singapore for supervising the field work of the Singapore Chinese Health Study. We also thank the Ministry of Health in Singapore for assistance with the identification of cancer cases via database linkages, and the National University Health System in Singapore for supporting the storage of biospecimens in the Singapore Chinese Health Study.

Funding National Institutes of Health (NCI R01 CA55069, R35 CA53890 and R01 CA80205). Johnson is a recipient of the training fellowship supported by the National Institutes of Health under Ruth L. Kirschstein National Research Service Award (T32CA132670).

\section{References}

1. Ferlay, J., et al. IARC cancerbase No. 5 version 2.0. International Agency for Research of Cancer; Lyon: 2004. GLOBOCAN 2002: cancer incidence, mortality, and prevalence worldwide.

2. Yu MC, Yuan JM. Environmental factors and risk for hepatocellular carcinoma. Gastroenterology. 2004; 127(5 Suppl 1):S72-S78. [PubMed: 15508106]

3. Azam S, et al. Antioxidant and prooxidant properties of caffeine, theobromine and xanthine. Med Sci Monit. 2003; 9(9):BR325-BR330. [PubMed: 12960921]

4. Okano J, et al. Caffeine inhibits the proliferation of liver cancer cells and activates the MEK/ERK/ EGFR signalling path-way. Basic Clin Pharmacol Toxicol. 2008; 102(6):543-551. [PubMed: 18346049]

5. Hosaka S, et al. Hepatocarcinogenesis inhibition by caffeine in ACI rats treated with 2acetylaminofluorene. Food Chem Toxicol. 2001; 39(6):557-561. [PubMed: 11346485]

6. Nakanishi N, et al. Effects of coffee consumption against the development of liver dysfunction: a 4year follow-up study of middle-aged Japanese male office workers. Ind Health. 2000; 38(1):99-102. [PubMed: 10680318]

7. Gross G, Jaccaud E, Huggett AC. Analysis of the content of the diterpenes cafestol and kahweol in coffee brews. Food Chem Toxicol. 1997; 35(6):547-554. [PubMed: 9225012]

8. Cavin C, et al. Protective effects of coffee diterpenes against aflatoxin B1-induced genotoxicity: mechanisms in rat and human cells. Food Chem Toxicol. 2001; 39(6):549-556. [PubMed: 11346484]

9. Eaton DL, Gallagher EP. Mechanisms of aflatoxin carcinogenesis. Annu Rev Pharmacol Toxicol. 1994; 34:135-172. [PubMed: 8042848]

10. Cavin C, et al. Cafestol and kahweol, two coffee specific diterpenes with anticarcinogenic activity. Food Chem Toxicol. 2002; 40(8):1155-1163. [PubMed: 12067578]

11. Gallus S, et al. Does coffee protect against hepatocellular carcinoma? Br J Cancer. 2002; 87(9): 956-959. [PubMed: 12434283]

12. Gelatti U, et al. Coffee consumption reduces the risk of hepatocellular carcinoma independently of its aetiology: a case-control study. J Hepatol. 2005; 42(4):528-534. [PubMed: 15868652]

13. Inoue $\mathrm{M}$, et al. Influence of coffee drinking on subsequent risk of hepatocellular carcinoma: a prospective study in Japan. J Natl Cancer Inst. 2005; 97(4):293-300. [PubMed: 15713964]

14. Kurozawa $Y$, et al. Coffee and risk of death from hepatocellular carcinoma in a large cohort study in Japan. Br J Cancer. 2005; 93(5):607-610. [PubMed: 16091758]

15. Montella $\mathrm{M}$, et al. Coffee and tea consumption and risk of hepatocellular carcinoma in Italy. Int $\mathbf{J}$ Cancer. 2007; 120(7):1555-1559. [PubMed: 17205531]

16. Shimazu T, et al. Coffee consumption and the risk of primary liver cancer: pooled analysis of two prospective studies in Japan. Int J Cancer. 2005; 116(1):150-154. [PubMed: 15756689]

17. Tanaka K, et al. Inverse association between coffee drinking and the risk of hepatocellular carcinoma: a case-control study in Japan. Cancer Sci. 2007; 98(2):214-218. [PubMed: 17233838]

18. Wakai $\mathrm{K}$, et al. Liver cancer risk, coffee, and hepatitis $\mathrm{C}$ virus infection: a nested case-control study in Japan. Br J Cancer. 2007; 97(3):426-428. [PubMed: 17637681]

19. Larsson SC, Wolk A. Coffee consumption and risk of liver cancer: a meta-analysis. Gastroenterology. 2007; 132(5):1740-1745. [PubMed: 17484871] 
20. Bravi F, et al. Coffee drinking and hepatocellular carcinoma risk: a meta-analysis. Hepatology. 2007; 46(2):430-435. [PubMed: 17580359]

21. Parkin, DM.; Whelan, S.; Ferlay, J.; Storm, H. Cancer incidence in five continents Vol 1 to VIII. Lyon: 2005. IARC CancerBase No. 7

22. Hankin JH, et al. Singapore Chinese Health Study: development, validation, and calibration of the quantitative food frequency questionnaire. Nutr Cancer. 2001; 39(2):187-195. [PubMed: 11759279]

23. Seow A, et al. Urinary total isothiocyanate (ITC) in a population-based sample of middle-aged and older Chinese in Singapore: relationship with dietary total ITC and glutathione S-transferase M1/ T1/P1 genotypes. Cancer Epidemiol Biomarkers Prev. 1998; 7(9):775-781. [PubMed: 9752985]

24. Parkin DM. Global cancer statistics in the year 2000. Lancet Oncol. 2001; 2(9):533-543. [PubMed: 11905707]

25. Nieters A, et al. Effect of cytokine genotypes on the hepatitis B virus-hepatocellular carcinoma association. Cancer. 2005; 103(4):740-748. [PubMed: 15643599]

26. Breslow NE, Day NE. Statistical methods in cancer research. Volume I-The analysis of casecontrol studies. IARC Sci Publ. 1980; 32:5-338. [PubMed: 7216345]

27. Ruhl CE, Everhart JE. Coffee and tea consumption are associated with a lower incidence of chronic liver disease in the United States. Gastroenterology. 2005; 129(6):1928-1936. [PubMed: 16344061]

28. Klatsky AL, Armstrong MA. Alcohol, smoking, coffee, and cirrhosis. Am J Epidemiol. 1992; 136(10):1248-1257. [PubMed: 1476147]

29. Cavin C, et al. Induction of Nrf2-mediated cellular defenses and alteration of phase I activities as mechanisms of chemoprotective effects of coffee in the liver. Food Chem Toxicol. 2008; 46(4): 1239-1248. [PubMed: 17976884]

30. Cavin C, et al. The coffee-specific diterpenes cafestol and kahweol protect against aflatoxin B1induced genotoxicity through a dual mechanism. Carcinogenesis. 1998; 19(8):1369-1375. [PubMed: 9744531]

31. Scholl P, et al. Molecular biomarkers for aflatoxins and their application to human liver cancer. Pharmacogenetics. 1995; 5(Spec No):S171-S176. [PubMed: 7581490]

32. Peterson $\mathrm{S}$, et al. Coffee intake and risk of colorectal cancer among Chinese in Singapore: the Singapore Chinese Health Study. Nutr Cancer. 2010; 62(1):21-29. [PubMed: 20043256]

33. Feng R, et al. Inhibition of activator protein-1, NF-kappaB, and MAPKs and induction of phase 2 detoxifying enzyme activity by chlorogenic acid. J Biol Chem. 2005; 280(30):27888-27895. [PubMed: 15944151]

34. Mukhtar M, et al. Anti-human immunodeficiency virus type 1 gene therapy in human central nervous system-based cells: an initial approach against a potential viral reservoir. Hum Gene Ther. 2000; 11(2):347-359. [PubMed: 10680847]

35. Erba D, et al. Effectiveness of moderate green tea consumption on antioxidative status and plasma lipid profile in humans. J Nutr Biochem. 2005; 16(3):144-149. [PubMed: 15741048]

36. Ui A, et al. Green tea consumption and the risk of liver cancer in Japan: the Ohsaki Cohort study. Cancer Causes Control. 2009; 20(10):1939-1945. [PubMed: 19768563]

37. Inoue $\mathrm{M}$, et al. Effect of coffee and green tea consumption on the risk of liver cancer: cohort analysis by hepatitis virus infection status. Cancer Epidemiol Biomarkers Prev. 2009; 18(6):17461753. [PubMed: 19505908]

38. Kurozawa Y, et al. Dietary habits and risk of death due to hepatocellular carcinoma in a large scale cohort study in Japan. Univariate analysis of JACC study data. Kurume Med J. 2004; 51(2):141149. [PubMed: 15373231]

39. Nagano J, et al. A prospective study of green tea consumption and cancer incidence, Hiroshima and Nagasaki (Japan). Cancer Causes Control. 2001; 12(6):501-508. [PubMed: 11519758] 


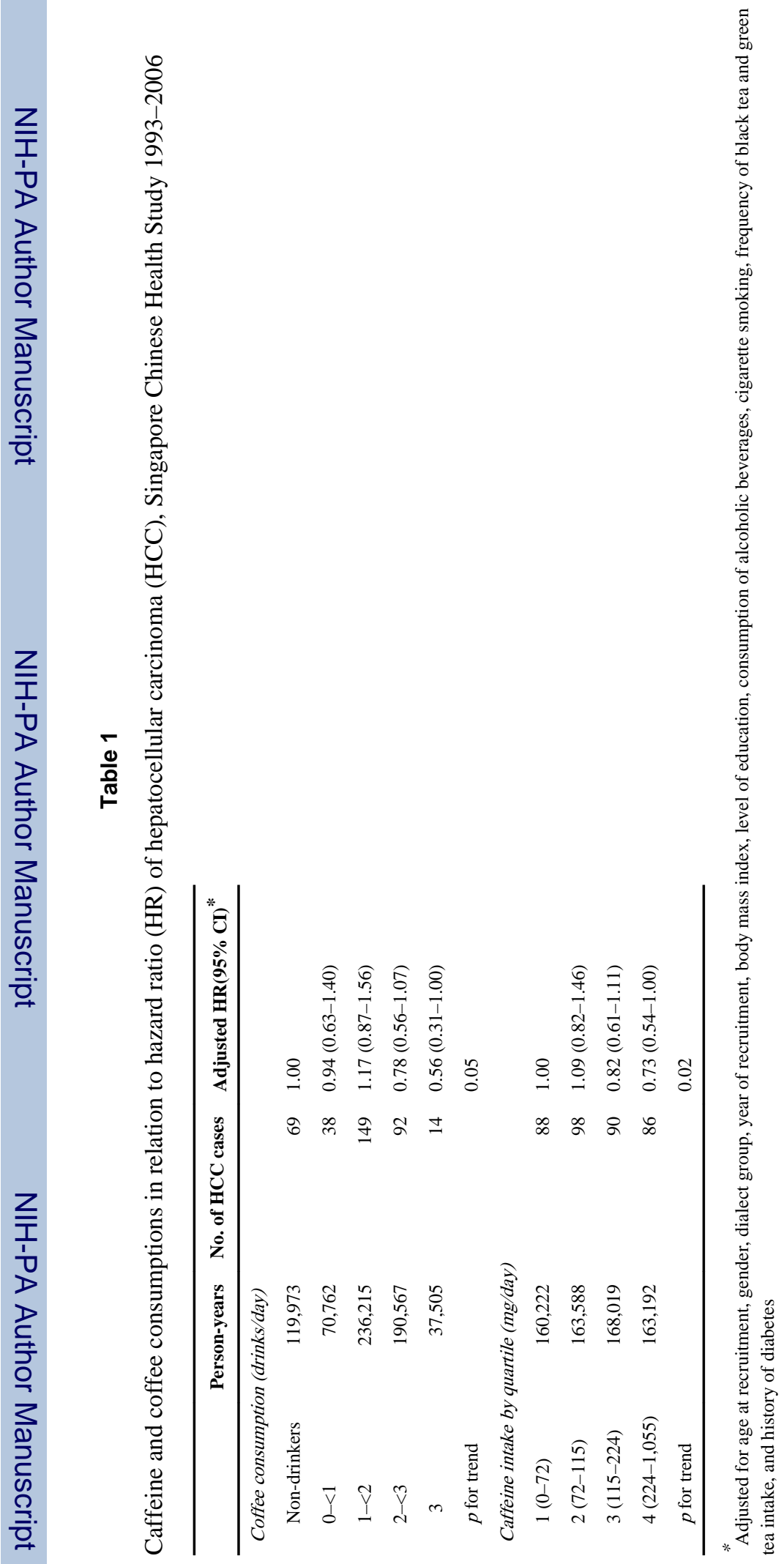

Cancer Causes Control. Author manuscript; available in PMC 2012 July 19. 


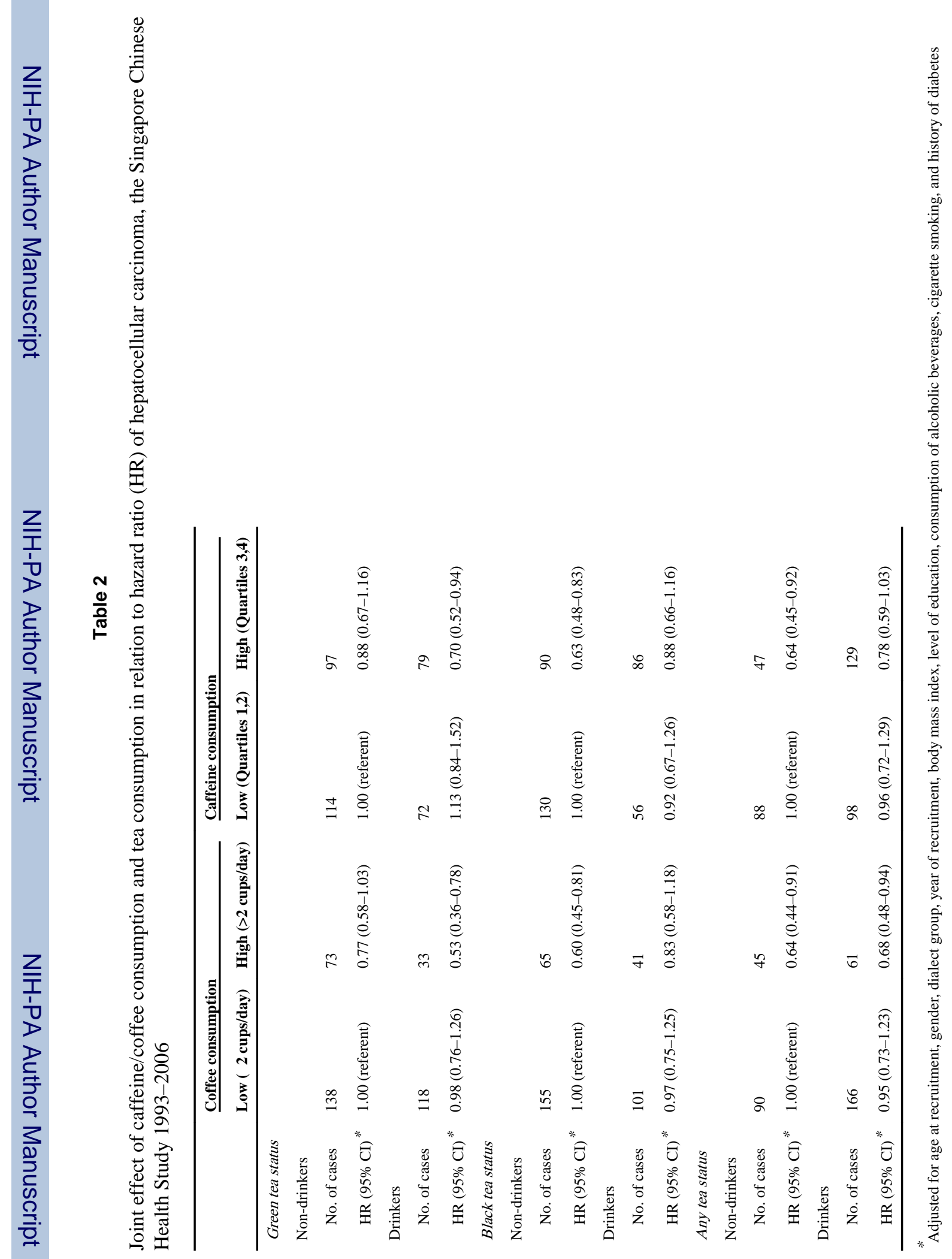




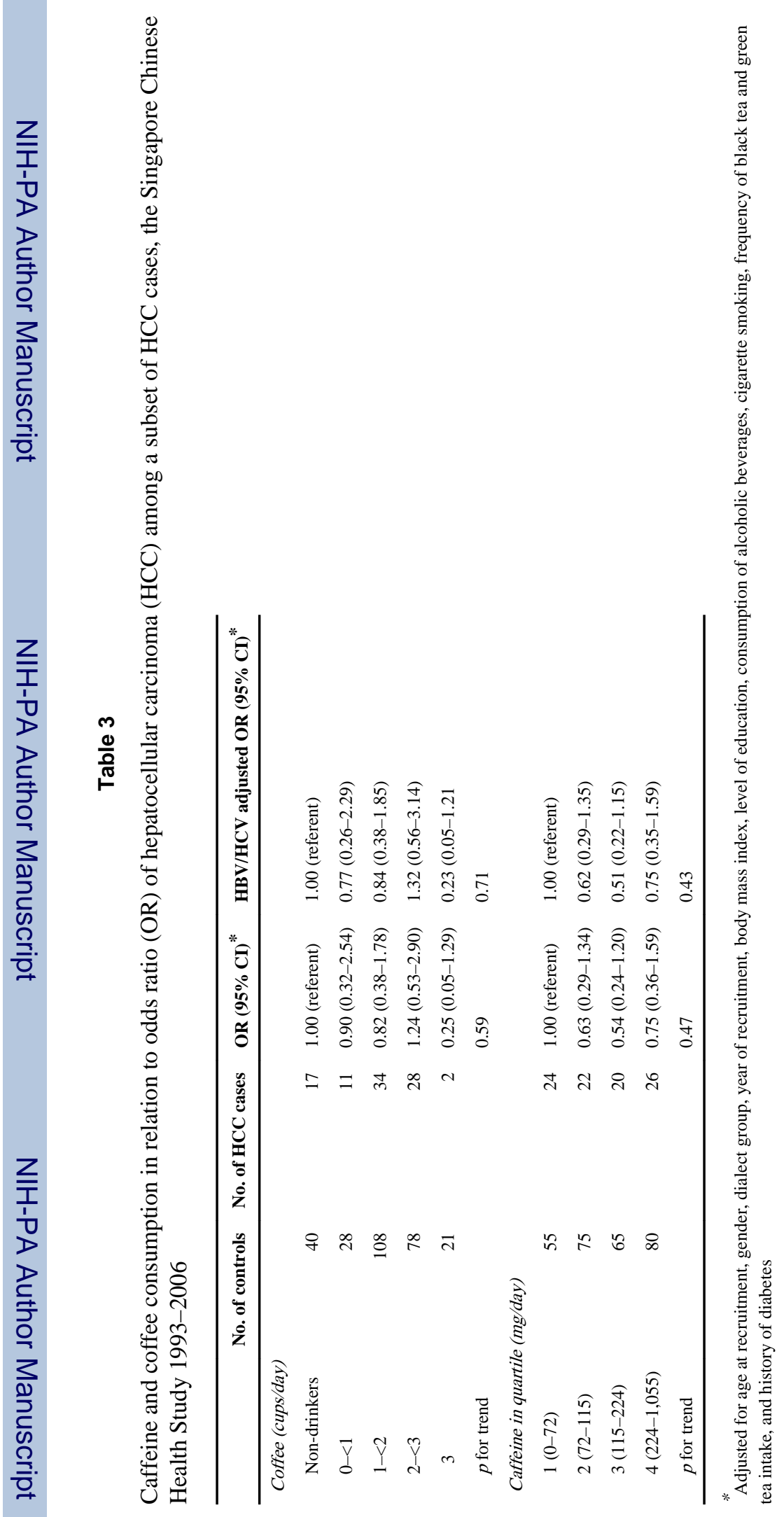

Cancer Causes Control. Author manuscript; available in PMC 2012 July 19. 